\title{
Analysis of metallic intermediate-band formation in photovoltaic materials
}

Cite as: Appl. Phys. Lett. 82, 151 (2003); https://doi.org/10.1063/1.1535744

Submitted: 06 June 2002 . Accepted: 15 November 2002 . Published Online: 30 December 2002

C. Tablero, and P. Wahnón

\section{ARTICLES YOU MAY BE INTERESTED IN}

Energetics of formation of $\mathrm{TiGa}_{3} \mathrm{As}_{4}$ and $\mathrm{TiGa}_{3} P_{4}$ intermediate band materials

The Journal of Chemical Physics 124, 014711 (2006); https://doi.org/10.1063/1.2140695

Intermediate band solar cells: Recent progress and future directions

Applied Physics Reviews 2, 021302 (2015); https://doi.org/10.1063/1.4916561

Detailed Balance Limit of Efficiency of p-n Junction Solar Cells

Journal of Applied Physics 32, 510 (1961); https://doi.org/10.1063/1.1736034

Lock-in Amplifiers Find out more today

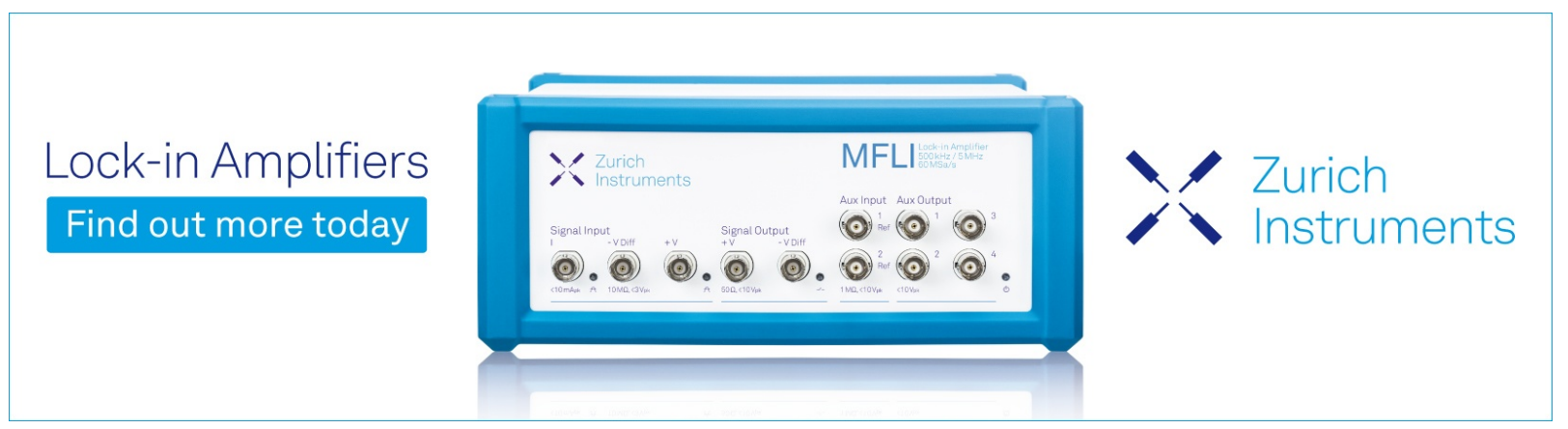

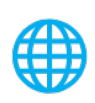

View Online 


\title{
Analysis of metallic intermediate-band formation in photovoltaic materials
}

\author{
C. Tablero a) and P. Wahnón \\ Instituto de Energía Solar, ETSI Telecomunicación, Universidad Politécnica de Madrid, \\ 28040 Madrid, Spain
}

(Received 6 June 2002; accepted 15 November 2002)

\begin{abstract}
The substitution of some transition atoms in III-V-type semiconductors may give rise to a type of high-efficiency photovoltaic material with an isolated intermediate narrow band in the middle of the band gap, capable of absorbing photons of low energies. We have carried out a comparative analysis of the nature and possibility of formation of the intermediate band in two compounds $\mathrm{Ga}_{4} \mathrm{P}_{3} \mathrm{Ti}$ and $\mathrm{Ga}_{3} \mathrm{P}_{4} \mathrm{Ti}$ in terms of density of states, electronic density, and atomic and orbital populations. We found that the intermediate band is formed only in one of these compounds. (C) 2003 American Institute of Physics. [DOI: 10.1063/1.1535744]
\end{abstract}

During the last decade many attempts have been made to increase the efficiency of solar cells based on indirect bandgap semiconductors. In particular, Luque and Martí ${ }^{1}$ have demonstrated the possibility of increasing the efficiency of ideal semiconductor solar cells, exceeding the thermodynamic limits established by Shockley and Queisser. ${ }^{2}$ They proposed a material capable of absorbing photons with energies lower than the semiconductor band gap, using an intermediate band situated inside this band gap. In the intermediate band, some of their states must be empty or half-filled in order that an electron from the valence band can be promoted to this intermediate, from which then another electron can be excited to the conduction band. In this way, two photons of low energy could produce an electron-hole pair of high free energy. ${ }^{3}$ In a recent work, ${ }^{4}$ using first-principle calculations, we have identified a kind of semiconductor compound that is a candidate for a high-efficiency photovoltaic material. These compounds of the type $\mathrm{Ga}_{4} X_{3} M$ with $X=$ As or $\mathrm{P}$, and $M$ $=$ transition metal, are formed by substituting some atoms in several III-V semiconductors with transition metal atoms. Some of these compounds show an intermediate, isolated, narrow band in the middle of the semiconductor band gap. Several factors such as width values of the intermediate band and the two band gaps, photon absorption coefficients or nonradiative recombination must account for the effectiveness of the processes. ${ }^{5}$

The main purpose of the present work is to carry out a detailed comparison of two compounds, namely $\mathrm{Ga}_{4} \mathrm{P}_{3} \mathrm{Ti}$ and $\mathrm{Ga}_{3} \mathrm{P}_{4} \mathrm{Ti}$, with apparently similar characteristics but different behaviors. We found from our calculations that one of them exhibits an isolated, half-filled intermediate band, whereas the other does not. We show that the states of the half-filled intermediate band originate mainly from the transition metal atom. We explain these results in terms of bonding, density of states (DOS), and characteristics of the atomic and orbital populations.

We use Density Functional Theory first-principles calculations ${ }^{6}$ to analyze the intermediate band formation and the microscopic properties of the semiconductor compounds in bulk conditions. The calculations have been done with the

${ }^{a)}$ Electronic mail: ctablero@etsit.upm.es
SIESTA code, ${ }^{7}$ using the generalized gradient approximation (GGA) of Perdew, Burke, and Ernzerhof. ${ }^{8}$

We have used the nonlocal pseudopotential approach. It has been constructed using the Troullier-Martins scheme ${ }^{9}$ and Kleiman-Bylander ${ }^{10}$ factorization. The reference configurations used in this work have been $4 s^{2} 4 p^{0} 3 d^{2}$ for Ti, $4 s^{2} 4 p^{3} 4 d^{0}$ for As, $3 s^{2} 3 p^{3} 3 d^{0}$ for $\mathrm{P}$, and $4 s^{2} 4 p^{1} 4 d^{0}$ for $\mathrm{Ga}$. We have tested them over a range of cutoff radii.

The code uses a numerical implementation of the pseudoatomic basis set. ${ }^{11}$ Our calculations were carried out specifically for two systems, $\mathrm{Ga}_{4} \mathrm{P}_{3} \mathrm{Ti}$ and $\mathrm{Ga}_{3} \mathrm{P}_{4} \mathrm{Ti}$, with an eight-atom simple cubic unit cell and a set of atomic orbital bases of double $\zeta$ plus polarization quality for all the atoms.

We have carried out a comparative study of both systems in order to explain the formation of the intermediate band, including an analysis of energy band structure, effect of the basis set, $k$-point mesh, and structural parameters.

The initial crystalline structure used in this letter for both $\mathrm{Ga}_{4} \mathrm{P}_{3} \mathrm{Ti}$ and $\mathrm{Ga}_{3} \mathrm{P}_{4} \mathrm{Ti}$ are the $\mathrm{GaP}$ zinc-blende structure, where we have substituted either a $\mathrm{P}$ or a $\mathrm{Ga}$ atom with $\mathrm{Ti}$ (one hole or one electron more, respectively). However, it is worth noting that in all our calculations, the crystalline structure parameters are obtained by a minimization of the total energy relaxing the atomic positions and the unit cell. In our case, the residual atomic forces were less than $0.04 \mathrm{eV} \mathrm{A}^{-1}$ and the stress tolerance was less than $0.1 \mathrm{GPa}$. We have also studied a cubic cell, but with a different lattice parameter and atomic positions, since lowering of symmetry due to the substitution leads to small distortions with respect of the initial positions of the zinc-blende structure. In the $\mathrm{Ga}_{3} \mathrm{P}_{4} \mathrm{Ti}$ structure, the $\mathrm{Ti}$ is surrounded by four $\mathrm{P}$, the $\mathrm{Ga}$ by four $\mathrm{P}$, whereas $\mathrm{P}$ is surrounded by one $\mathrm{Ti}$ and three $\mathrm{Ga}$. In the $\mathrm{Ga}_{4} \mathrm{P}_{3} \mathrm{Ti}$ structure, the $\mathrm{Ti}$ is surrounded by four $\mathrm{Ga}$, the $\mathrm{P}$ by four $\mathrm{Ga}$, and the $\mathrm{Ga}$ by one $\mathrm{Ti}$ and three $\mathrm{P}$. The $a b$ initio energy band diagrams the two compounds $\mathrm{Ga}_{4} \mathrm{P}_{3} \mathrm{Ti}$ and $\mathrm{Ga}_{3} \mathrm{P}_{4} \mathrm{Ti}$ (Fig. 1), show that only the first exhibits an isolated, half-filled intermediate band that can absorb low-energy photons.

We now focus on the results obtained in the GGA corrections. The difference in electronic density between the total crystalline and the superposed atomic density $\Delta \rho=\rho$ $-\rho_{\text {atom }}$ is shown in Fig. 2 for the (110) plane. This difference 

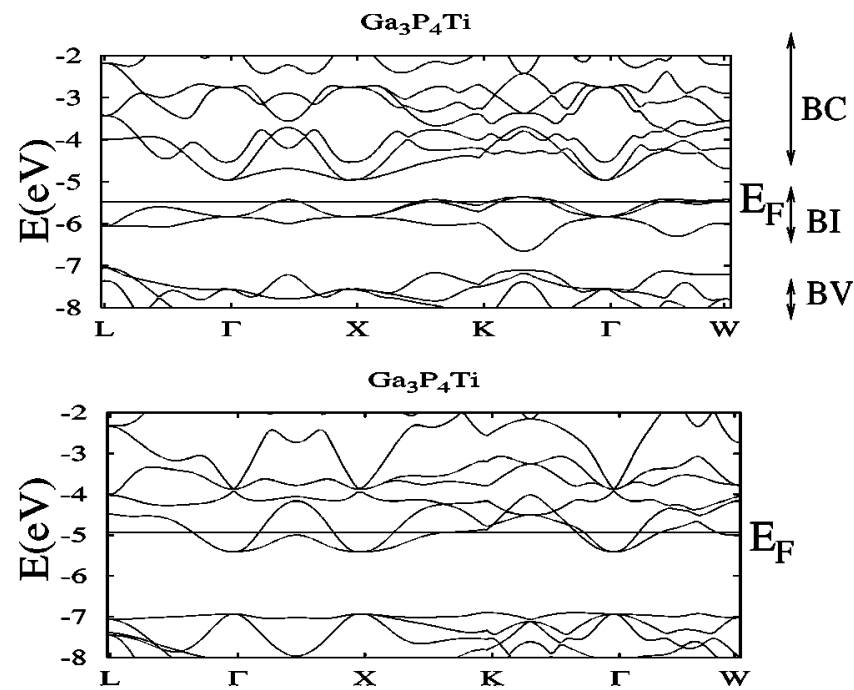

FIG. 1. Band dispersion for $\mathrm{Ga}_{4} \mathrm{P}_{3} \mathrm{Ti}$ and $\mathrm{Ga}_{3} \mathrm{P}_{4} \mathrm{Ti}$ in some directions of reciprocal space. In the figure, $\mathrm{BV}$ is the valence band, $\mathrm{BI}$ is the intermediate band, and $\mathrm{BC}$ is the conduction band.

can be positive or negative, and highlights the different behavior of the two compounds.

In Fig. 2, we observe a decrease in the charge density $(\Delta \rho<0)$, both for $\mathrm{Ga}_{3} \mathrm{P}_{4} \mathrm{Ti}$ and for $\mathrm{Ga}_{4} \mathrm{P}_{3}$ Ti in the nonbonding directions for the $\mathrm{Ga}$ and $\mathrm{P}$ atoms, and an increase in the charge density $(\Delta \rho>0)$ in the bonding directions of Ti-P and $\mathrm{P}-\mathrm{Ga}$. However, the behavior of the Ti atom in both compounds is quite different. For $\mathrm{Ga}_{3} \mathrm{P}_{4}$ Ti Fig. 2(a) shows a decrease in the charge density in the nonbonding directions corresponding to the $d_{x y}, d_{x z}$, and $d_{y z}$ orbitals, and an increase in the charge for these same orbitals, but in the Ti-P bonding direction, where a maximum of the charge density is exhibited. Figure 2(a), on the other hand, shows a nonbonding charge density increase in the $z$ direction around the $\mathrm{Ti}$ atom. In summary, we can say that the Ti $d_{x y}, d_{x z}$, and $d_{y z}$ orbitals make bonds with the corresponding $s p$ of the $\mathrm{P}$ atom, and that the other two Ti $d_{z^{2}}$ and $d_{x^{2}-y^{2}}$ orbitals are nonbonding and contain the remaining electronic charge.

The situation for the $\mathrm{Ga}_{4} \mathrm{P}_{3} \mathrm{Ti}$ compound is completely different, the substitution of one $\mathrm{P}$ atom by a Ti creates a hole related to the GaP host semiconductor. Figure 2(b) shows a significant decrease in the charge density of the Ti $d_{z^{2}}$ orbitals and an increase in the Ti-Ga direction, but without reach-

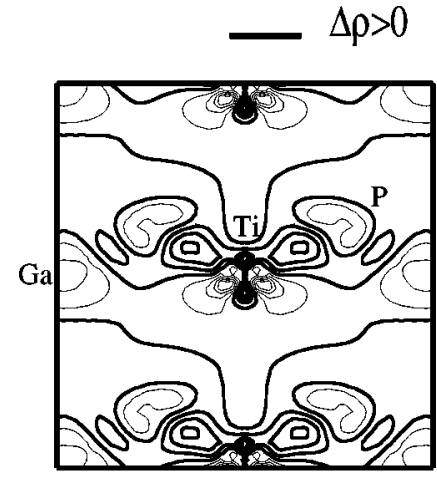

(a)

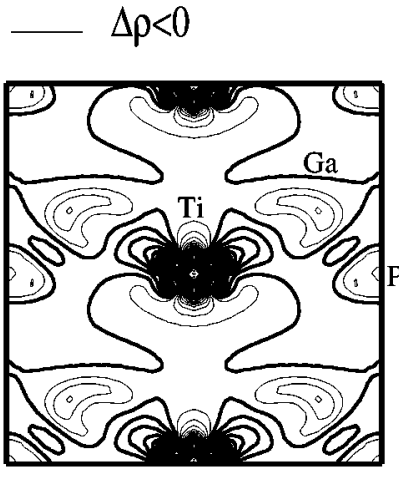

(b)
FIG. 2. Difference between the crystalline and superposed atomic charge density $\Delta \rho=\rho-\rho_{\text {atom }}$ at the (110) plane for (a) $\mathrm{Ga}_{3} \mathrm{P}_{4} \mathrm{Ti}$ and (b) $\mathrm{Ga}_{4} \mathrm{P}_{3} \mathrm{Ti}$.
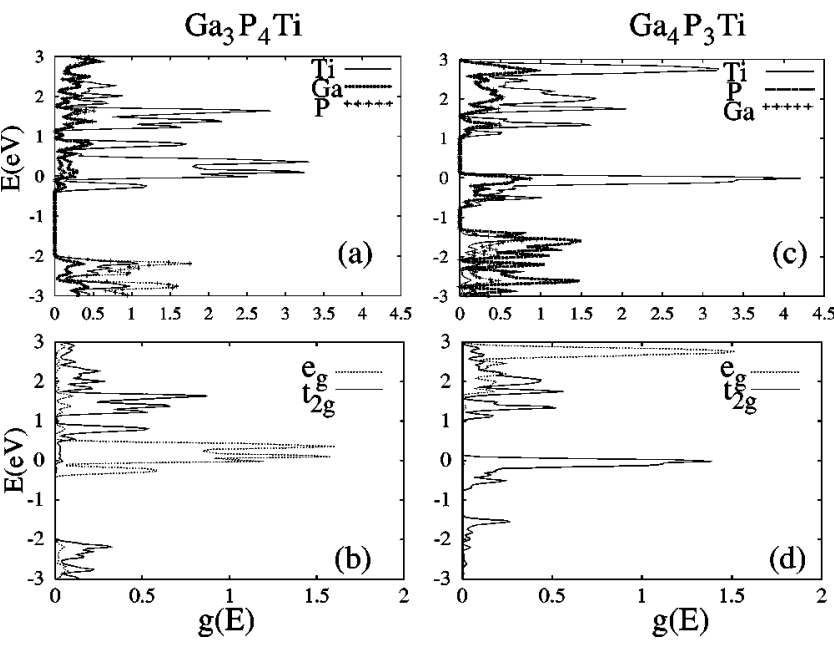

FIG. 3. (a) Projected DOS on each atom for $\mathrm{Ga}_{3} \mathrm{P}_{4}$ Ti. (b) Projected DOS on $t_{2 g}$-group $\left(d_{x y}, d_{x z}\right.$, and $\left.d_{y z}\right)$ and $e_{g}$-group $\left(d_{x^{2}-y^{2}}\right.$ and $\left.d_{z^{2}}\right)$ orbitals for $\mathrm{Ga}_{3} \mathrm{P}_{4}$ Ti. Similar legend for (c) and (d) for $\mathrm{Ga}_{4} \mathrm{P}_{3} \mathrm{Ti}$.

ing any maximum value. This charge accumulation, around the $\mathrm{Ti}$ and the $\mathrm{Ti}-\mathrm{Ga}$ direction is mainly a nonbonding charge, which means that the Ti does not really make a bond with the tetrahedric orbitals of Ga arranged around it.

In order to understand the formation of the isolated intermediate band in the semiconductor compounds and the atomic composition of their states, the total DOS and the projected DOS on atoms and in the $d$ orbitals of Ti have been calculated.

The DOS for $\mathrm{Ga}_{4} \mathrm{P}_{3} \mathrm{Ti}$ and $\mathrm{Ga}_{3} \mathrm{P}_{4} \mathrm{Ti}$ are shown in Fig. 3. The (a) and (c) panel of this figure summarize the partial DOS projected on each atom, and the (b) and (d) panels display the partial density projected on $d_{x y}, d_{x z}, d_{y z}$ and $d_{z^{2}}$, $d_{x^{2}-y^{2}}$ Ti orbitals.

Because of the approximate tetrahedric symmetry around the Ti atom that the crystalline structure exhibits, the $d$ orbitals break their degeneracy forming two groups. One component is formed by the $d_{z^{2}}$ and $d_{x^{2}-y^{2}}$ orbitals ( $e_{g}$-group) and the other by the $d_{x y}, d_{x z}$, and $d_{y z}$ $\left(t_{2 g}\right.$-group). The $t_{2 g}$-components are oriented along the directions of the first and second neighbors, and they make part of the bond when they are formed. However, the $e_{g}$-components will not form bonds because they do not have the appropriate symmetry. They are either empty or filled with nonbonding electrons.

A first analysis of Fig. 3(c) shows, in the case of the $\mathrm{Ga}_{4} \mathrm{P}_{3} \mathrm{Ti}$, a half-filled intermediate band near and below of the Fermi level (taken as zero energy), as well as the two energy gaps around it. This intermediate band presents a rather sharp peak, a characteristic of a narrow band. This band is formed mainly by the contribution of the Ti orbitals, as is shown in the figure. Moreover, it can be seen in the Fig. 3(d) that the intermediate band is formed mainly by orbitals of the $t_{2 g}$-group. An analysis of the wave function shows that the $p$ orbitals from the closest $\mathrm{P}$ atoms contribute very little to the formation of this intermediate band. The $e_{g}$-group orbitals of Ti [Fig. 3(d)] lie above the Fermi level and are empty.

In the case of the $\mathrm{Ga}_{3} \mathrm{P}_{4} \mathrm{Ti}$ system, no isolated intermediate band appears, although the atom that makes a main contribution to the DOS at the Fermi level is also the Ti 
TABLE I. Mulliken population analyses for $\mathrm{Ga}_{3} \mathrm{P}_{4} \mathrm{Ti}, \mathrm{Ga}_{4} \mathrm{P}_{3} \mathrm{Ti}$, and $\mathrm{Ga}_{4} \mathrm{P}_{4}$ systems. $p_{1}, p_{2}$, and $p_{3}$ are, alternately, the $p_{x}, p_{y}$, and $p_{z}$ orbitals.

\begin{tabular}{|c|c|c|c|c|}
\hline \multicolumn{5}{|c|}{$\mathrm{Ga}_{3} \mathrm{P}_{4} \mathrm{Ti}\left(Q_{\mathrm{tot}}=33.000\right)$} \\
\hline Atom & $Q_{\text {atom }}$ & $s$ & $t_{2 g}$ & $e_{g}$ \\
\hline $\mathrm{Ti}$ & 2.930 & 0.503 & 0.454 & 0.532 \\
\hline & & $s$ & $p_{1}, p_{2}$ & $p_{3}$ \\
\hline $\mathrm{Ga}$ & 2.857 & 1.285 & 0.533 & 0.506 \\
\hline $\mathrm{P}$ & 5.375 & 1.663 & 1.237 & 1.237 \\
\hline \multicolumn{5}{|c|}{$\mathrm{Ga}_{4} \mathrm{P}_{3} \mathrm{Ti} \quad\left(Q_{\text {tot }}=31.000\right)$} \\
\hline$Q_{\text {atom }}$ & $s$ & \multicolumn{2}{|c|}{$t_{2 g}$} & $e_{g}$ \\
\hline 2.940 & 0.558 & \multicolumn{2}{|c|}{0.778} & 0.024 \\
\hline & $s$ & \multicolumn{2}{|c|}{$p_{1}, p_{2}$} & $p_{3}$ \\
\hline 3.110 & 1.417 & \multicolumn{2}{|c|}{0.564} & 0.564 \\
\hline 5.206 & 1.670 & \multicolumn{2}{|c|}{1.169} & 1.199 \\
\hline \multicolumn{5}{|c|}{$\mathrm{Ga}_{4} \mathrm{P}_{4}\left(Q_{\mathrm{tot}}=32.000\right)$} \\
\hline Atom & $Q_{\text {atom }}$ & & & $p_{1}, p_{2}, p_{3}$ \\
\hline $\mathrm{Ga}$ & 2.741 & & & 0.528 \\
\hline $\mathrm{P}$ & 5.259 & & & 1.229 \\
\hline
\end{tabular}

atom. We note that some orbitals of the $t_{2 g}$-group are present near the Fermi level [Fig. 3(b)]. Therefore, some bonding between $t_{2 g}$-components and $p$ orbitals of the $\mathrm{P}$ are formed. However, the $e_{g}$-group [Fig. 3(b)] presents a rather sharp peak around the Fermi level and contains the nonbonding electronic charge in excess.

Mulliken analyses have been carried out in order to have a quantitative comparison for atoms and orbital populations. In Table I, we see that the atomic charge in Ti is very similar for both $\mathrm{Ga}_{3} \mathrm{P}_{4} \mathrm{Ti}$ and $\mathrm{Ga}_{4} \mathrm{P}_{3} \mathrm{Ti}$ systems. However, for the $t_{2 g}$-group of orbitals, the populations are very different in the two systems studied. In $\mathrm{Ga}_{3} \mathrm{P}_{4} \mathrm{Ti}$, the difference between the two groups is small. This result is consistent with the previous analysis of the electronic charge density and the DOS, where the orbitals of the $t_{2 g}$-group form bonds with the closest four tetrahedric $\mathrm{P}$ atoms, whereas the orbitals of the $e_{g}$-group have an electronic density with a nonbonding character. For $\mathrm{Ga}_{4} \mathrm{P}_{3} \mathrm{Ti}$, however, the associated electronic charge for the $e_{g}$-group is very small, and the $t_{2 g}$-group has a charge bigger than in the previous system. These last results, with no quasi bond between Ti-Ga, but very weak bonds with the second neighbors ( $\mathrm{P}$ atoms), are in accordance with the analysis carried out previously. Most of the charge of the $t_{2 g}$-group belongs to a nonbonding electronic density.

On the other hand, we can also see from the table that the atomic charge associated with $\mathrm{P}$ and $\mathrm{Ga}$ atoms is bigger in $\mathrm{Ga}_{3} \mathrm{P}_{4} \mathrm{Ti}$ than in the GaP host semiconductor. This is due mainly to the excess electrons of $\mathrm{Ga}_{3} \mathrm{P}_{4} \mathrm{Ti}$ compared to the cubic cell of GaP. However, the orbital population distribution in the $p$ orbitals of $\mathrm{Ga}$ and $\mathrm{P}$ are similar for both systems, which is consistent with the semiconductor property obtained for $\mathrm{Ga}_{3} \mathrm{P}_{4} \mathrm{Ti}$ in contrast to $\mathrm{Ga}_{4} \mathrm{P}_{3} \mathrm{Ti}$, for which the orbital population distribution is very different from $\mathrm{GaP}$. Moreover, in the case of $\mathrm{Ga}_{4} \mathrm{P}_{3} \mathrm{Ti}$, the atomic population of the $\mathrm{P}$ atom is smaller than in the $\mathrm{GaP}$ semiconductor, due mainly to the hole. However, the electronic charge on $\mathrm{Ga}$ is greater, due to the nonbonding formation with $\mathrm{Ti}$.

In conclusion, we have investigated the electronic properties of $\mathrm{Ga}_{4} \mathrm{P}_{3} \mathrm{Ti}$ and $\mathrm{Ga}_{3} \mathrm{P}_{4} \mathrm{Ti}$ in order to identify the nature and possibility of forming an isolated intermediate band in the middle of a host semiconductor band gap, a prerequisite for a high-efficiency photovoltaic material. For this task, we used an $a b$ initio periodic density functional method which is solved fully self-consistently with the local and semi-local density approximation. In our results, we found that it is theoretically possible to design new photovoltaic materials with an isolated intermediate band using a host semiconductor of the zinc-blende structure, but only in the case in which the transition metal Ti replaces one of the $\mathrm{P}$ atoms, but not one of the $\mathrm{Ga}$ atoms. In the former case, holes are formed in the intermediate band states and no bonds are formed between the Ga-Ti. Moreover, from our study, we can deduce that the substitute atom must have $d$ orbitals, which can form a bond with $\mathrm{P}$ to accommodate the nonbonding electrons of the system.

The authors want to thank Prof. A. Luque and Prof. A. Martí for fruitful discussions. This work was partially supported by Plan Nacional de I+D of Spain program Ref. TIC20000-1339-C02-02 and the European Commission Contract ENK6 CT200 00310.

\footnotetext{
${ }^{1}$ A. Luque and A. Martí, Phys. Rev. Lett. 78, 5014 (1997); Prog. Photovoltaics 9, 73 (2001).

${ }^{2}$ W. Shockley and H. Queisser, J. Appl. Phys. 32, 510 (1961).

${ }^{3}$ A. Luque, F. Flores, A. Martí, J. C. Conesa, P. Wahnón, J. Ortega, C. Tablero, R. Pérez, and L. Cuadra, Patent No. WO 00/77829, Int. Bureau of WIPO, (21 Dec. 2000).

${ }^{4}$ P. Wahnón and C. Tablero, Phys. Rev. B 65, 165115 (2002).

${ }^{5}$ C. Tablero, P. Wahnón, L. Cuadra, A. Martí, J. Fernández, and A. Luque, Proceedings of the 17th European Photovoltaic Solar Energy Conference, Munich, 2001, Vol. I, pp. 296-299 (ISBN 3-936338-08-6).

${ }^{6}$ W. Kohn and L. J. Sham, Phys. Rev. 140, 1133 (1965).

${ }^{7}$ E. Artacho, D. Sánchez-Portal, P. Ordejón, A. García, and J. M. Soler, Phys. Status Solidi B 215, 809 (1999), and references therein.

${ }^{8}$ J. P. Perdew, K. Burke, and M. Ernzerhof, Phys. Rev. Lett. 77, 3865 (1996); 78, 1396 (1997).

${ }^{9}$ N. Trouiller and J. L. Martins, Phys. Rev. B 43, 1993 (1991).

${ }^{10}$ L. Kleinman and D. M. Bylander, Phys. Rev. Lett. 48, 1425 (1982); D. M. Bylander and L. Kleinman, Phys. Rev. B 41, 907 (1990).

${ }^{11}$ O. F. Sankey and D. J. Niklewski, Phys. Rev. B 40, 3979 (1989).
} 